\title{
Categorization of Single Degree of Freedom Eight Link Kinematic Chains and Determination of Automorphism
}

\author{
Dr. Syed Shane Haider Rizvi' ${ }^{1}$ and Javed Kaleem ${ }^{2}$ \\ ${ }^{1,2}$ Assistnat Professor, Mechanical Engineering Section, University Polytechnic, Jamia Millia Islamia, New Delhi , India.
}

\begin{abstract}
Human civilization right from the stone age requires machines for their sustenance on the earth, the early age stone tools or the discovery of the wheel was the landmarks in the history of civilization but as the human race develops more and more complex machineries comes into existence and the basis of all these machines are the kinematic chains. The present study is to look into the available categorization of these kinematic chains in the literature and to develop a new and effective method of categorization of the available non-isomorphic single degree of freedom eight link chains and to find the automorphs present in each chain.
\end{abstract}

Keywords: Automorphism, degree of freedom, kinematic chains.

\section{INTRODUCTION}

Professor ALT (1953) published a collection of these ten bar chains, consisting of 222 varieties. While compiling the collection, there was a problem to separate the two arrangements, which might look alike, were the same or different. Thus a new term among linkages is introduced i.e. isomorphism between linkages. Franke (1958)[1] developed the categorization of kinematic chains by representing the links in the form of circles and bands each polygonal link is shown by a circles associated with a number that will be equal to the number of pairs formed by the links in connection with other links and each band associated with a number other than zero represents a binary link, a band associated with number zero shows a direct connection between the two polygonal link. This classification shows the chains as molecules and chains with similar properties looks alike. In 1964 it was found that ALT's work was avilable in the files of the Lehrstuhl fuer Foerderund Getriebetechnik. ALT's work consists of thirty one sheets drawings on which he has made a collection of ten link planar chains, as ALT's work was similar to that of Franke (1958)[1], now the ten link 1 dof chains increases to 230 . He primarily takes up such patterns only which are made by all members leaving the binary links, and are arranged in groups from I to VII, based on the number, arrangement and size of each of the constituent link, further these were subdivided into 'subgroups' on the basis of their connective patterns. ALT had not assumed that the length of the connecting path between the larger bodies with binary chains can have a length of less than one (i.e. one binary member and two joints.). But Franke on the contrary took zero (i.e. One joint only) as the minimum length of the path.. Crossley (1966) [2] proposed a collection of 10-link plane chains. He compares this collection with the results of Professor ALT (1953) and raised the number of the invariants to 230. Reuleaux[3] introduced a way of representing the kinematic relations through symbols for planer and spatial mechanisms and the symbols look like molecules, kinematic chains with similar molecule representation are put in same group. Mruthyunjaya (1984) [4, 5, and 6] presented a computerized methodology for structural synthesis of kinematic chains: part-1, part-2 and part-3. In part-1, he made an effort to develop a fully computerized approach for structural synthesis of kinematic chains. The various steps involved in the method of structural synthesis are based on transformation of binary chains. Mathematical modelling for structural analysis is done to develop a computer program for structural synthesis and analysis of kinematic chains with simple joints. In part-2, a computer program developed in part-1 is checked for the reliability and it has been proved by pplying the program to several cases, which are either fully or partially not available in the literature, such as 8- and 10-link, single -degree of freedom chains; 12-link, single -degree of freedom binary chains; and 9-link, two-degree of freedom chains. By the use of above process, errors in the results of other researchers have been reported. In part-3 the method is applied to the 10 link 3 dof chains. Agrawal and Rao (1987) [8] introduced loop freedom matrix and its permanent function on the mobility properties of kinematic chains and develops a systematic method of analysis of the mobility properties of the kinematic chains. They also introduced a method to find degree of freedom (DOF) of any mechanism having constraints may be constant or variable, having simple or multiple joints. Sethi and Agrawal (1993) [9] presented a hierarchical classification of kinematic chains by using a multigraph approach. They classify the kinematic chains into different categories on the basis of structural properties, for selecting the best basic structure of a mechanism. Mathematical modelling is done with the Franke's condensed notations by a multigraph and variable permanent matrix. A 6-step hierarchical classification scheme was developed for the various invariants of a multigraph, to classify families of kinematic chains and it also segregates the kinematic chains having similar sub-sets of structures forming isomorphic subchains.

Ting and Dou (1996) [10] worked on classification and branch identification of Stephenson six bar chains. They showed that a Stephenson six bar chain consists a four bar loop and a five bar loop, rotatability and the interaction of both loops affects its branch condition According to this paper, A Stephenson 
chain can have up to six branches and the condition of branches is more complicated than the branches of a four bar chain. In this paper, a method to identify the effects of both loops on the rotatability of any Stephenson six bar linkage and the algorithms to check its branch condition are developed.

\section{METHODOLOGY OF CATEGORIZATION}

A Chain Identification (CI) matrix of a kinematic chain is a unique representation that defines a chain completely, but before obtaining the matrix the following definitions should be kept in mind [11].

Link - Each component of the kinematic chain is a single link provided that it has a relative motion with the other, but if two components are rigidly fixed they are not considered as two different links they are treated as a single link. The type of the link depends upon the connections they have with the other links if a link is connected with two other links then it is a binary link, if it has three connections then it is termed as a ternary link and so on.

Degree of a Link - the degree of a binary link is 2, ternary link is 3 and the quaternary link is 4 and so on

CI matrix of the selected kinematic chain is obtained as explained below

$>\mathrm{CI}_{\mathrm{i}, \mathrm{j}}=0$ if $\mathrm{i}^{\text {th }}$ link is connected to $\mathrm{j}^{\text {th }}$ link

$>\mathrm{CI}_{\mathrm{i}, \mathrm{j}}=\mathrm{n}$, where ' $\mathrm{n}$ ' is the number of links commonly connected to both $\mathrm{i}^{\text {th }}$ and $\mathrm{j}^{\text {th }}$ links

$>\mathrm{CI}_{\mathrm{i}, \mathrm{j}}=$ degree of that link, if $\mathrm{i}=\mathrm{j}$,

$>\mathrm{CI}_{\mathrm{i}, \mathrm{j}}=0$ if $\mathrm{i}^{\text {th }}$ link and $\mathrm{j}^{\text {th }}$ link are not commonly connected to any other link of the chain.

A unique number which classifies the planar kinematic chains, according to the link assortment of the kinematic chain, chains with same link assortment have a common GIDN. The GIDN of a chain can be obtained by the product of the highest degree value of the links of the chain and the sum of all the elements of the CI matrix of the chain.

\section{DETERMINATION OF AUTOMORPHISM}

While developing the mechanisms of a kinematic chain, there is chance of getting similar mechanisms from the different links of a kinematic chain if they are chosen as frame. There may be one or more pairs of similar mechanisms. This phenomenon is known as Automorphism.

The CI matrix is used to determine the invariant LSI for each link of the chain the similar values of LSI determines the similar mechanisms. The procedure of finding the invariant LSI is as follows.

1. Generate the CI matrix as explained in the section II

2. Choose the link to be fixed for obtaining the mechanism
3. Replace the diagonal element of the CI matrix with zero for the link which is made to fix.

4. Obtain the Eigen values of the matrix obtained in step-3.

5. Determine the link invariant LSI by summing the absolute Eigen values.

6. Repeat the procedure from step-2 to step-5 by choosing another link until all links are used.

7. Now compare the LSI values, similar values indicate similar mechanisms.

8. Count the number of pairs of automorphs from similar LSI values.

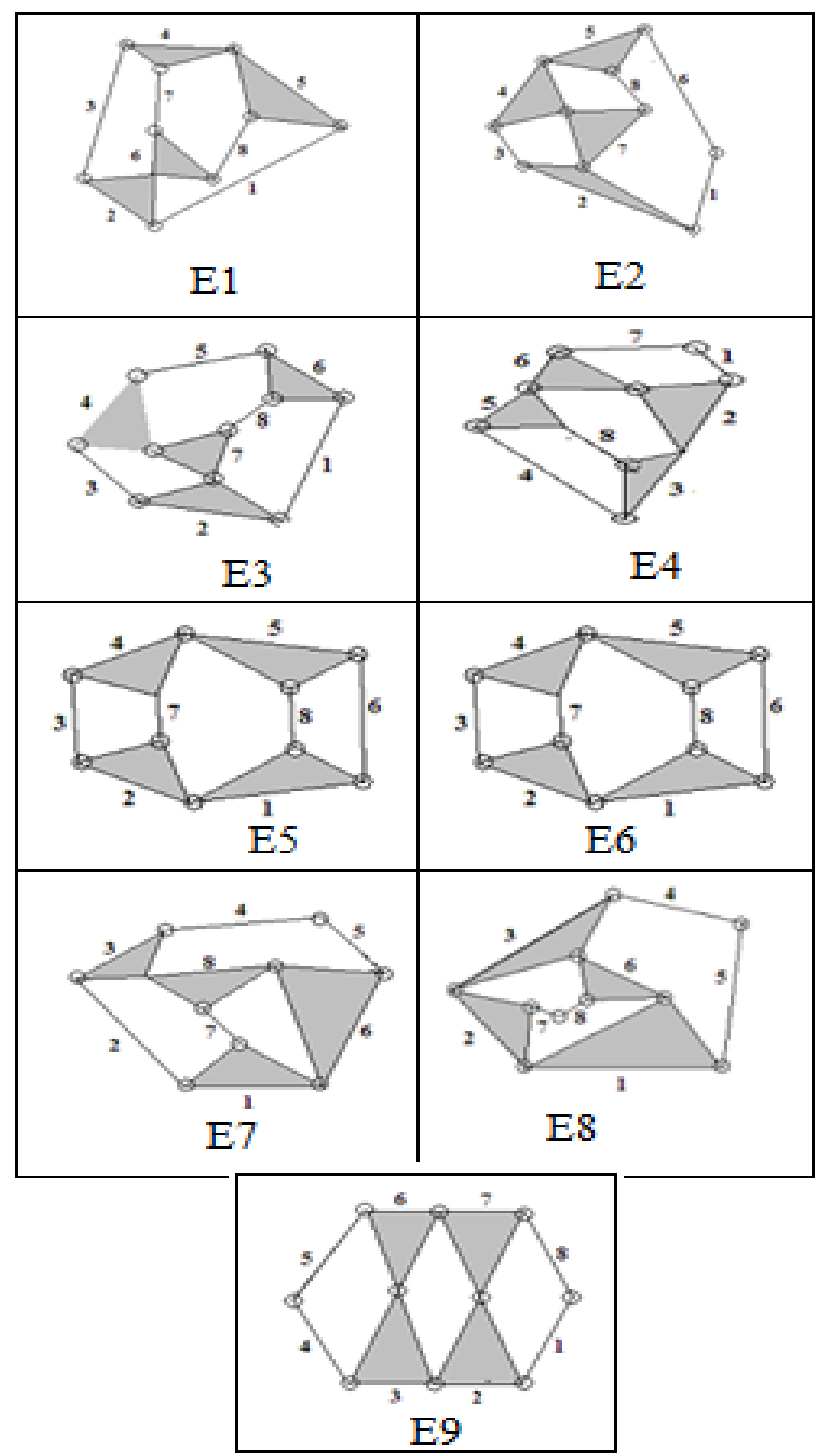

Fig.1. Eight link chains with assortment [2(4)-3(4)]

Fig1, contains the single degree of freedom chains with eight links all having 4 binary and 4 ternary links hence the link assortment of all the chains is [2(4)-3(4)]. 


\section{APPLICATION TO KINEMATIC CHAINS}

CI matrices for the chains from E1 to E9 are written below using the method explained in the section II

$\mathrm{CIE} 1=\left|\begin{array}{llllllll}2 & 0 & 1 & 1 & 0 & 1 & 0 & 1 \\ 0 & 3 & 0 & 1 & 1 & 0 & 1 & 1 \\ 1 & 0 & 2 & 0 & 1 & 1 & 1 & 0 \\ 1 & 1 & 0 & 3 & 0 & 1 & 0 & 1 \\ 0 & 1 & 1 & 0 & 3 & 1 & 1 & 0 \\ 1 & 0 & 1 & 1 & 1 & 3 & 0 & 0 \\ 0 & 1 & 1 & 0 & 1 & 0 & 2 & 1 \\ 1 & 1 & 0 & 1 & 0 & 0 & 1 & 2\end{array}\right|$

$\mathrm{CIE} 2=\left|\begin{array}{llllllll}2 & 0 & 1 & 0 & 1 & 0 & 1 & 0 \\ 0 & 3 & 0 & 2 & 0 & 1 & 0 & 1 \\ 1 & 0 & 2 & 0 & 1 & 0 & 2 & 0 \\ 0 & 2 & 0 & 3 & 0 & 1 & 0 & 2 \\ 1 & 0 & 1 & 0 & 3 & 0 & 2 & 0 \\ 0 & 1 & 0 & 1 & 0 & 2 & 0 & 1 \\ 1 & 0 & 2 & 0 & 2 & 0 & 3 & 0 \\ 0 & 1 & 0 & 2 & 0 & 1 & 0 & 2\end{array}\right|$

$\mathrm{CIE} 3=\left|\begin{array}{llllllll}2 & 0 & 1 & 0 & 1 & 0 & 1 & 1 \\ 0 & 3 & 0 & 2 & 0 & 1 & 0 & 1 \\ 1 & 0 & 2 & 0 & 1 & 0 & 2 & 0 \\ 0 & 2 & 0 & 3 & 0 & 1 & 0 & 1 \\ 1 & 0 & 1 & 0 & 2 & 0 & 1 & 1 \\ 0 & 1 & 0 & 1 & 0 & 3 & 1 & 0 \\ 1 & 0 & 2 & 0 & 1 & 1 & 3 & 0 \\ 1 & 1 & 0 & 1 & 1 & 0 & 0 & 2\end{array}\right| \quad \operatorname{CIE} 4=\left|\begin{array}{llllllll}2 & 0 & 1 & 0 & 0 & 2 & 0 & 0 \\ 0 & 3 & 0 & 1 & 1 & 0 & 2 & 1 \\ 1 & 0 & 3 & 0 & 2 & 1 & 0 & 0 \\ 0 & 1 & 0 & 2 & 0 & 1 & 0 & 2 \\ 0 & 1 & 2 & 0 & 3 & 0 & 1 & 0 \\ 2 & 0 & 1 & 1 & 0 & 3 & 0 & 1 \\ 0 & 2 & 0 & 0 & 1 & 0 & 2 & 0 \\ 0 & 1 & 0 & 2 & 0 & 1 & 0 & 2\end{array}\right|$

CIE5 $=\left|\begin{array}{llllllll}3 & 0 & 1 & 0 & 2 & 0 & 1 & 0 \\ 0 & 3 & 0 & 3 & 0 & 1 & 0 & 1 \\ 1 & 0 & 2 & 0 & 1 & 0 & 2 & 0 \\ 0 & 2 & 0 & 3 & 0 & 1 & 0 & 1 \\ 2 & 0 & 1 & 0 & 3 & 0 & 1 & 0 \\ 0 & 1 & 0 & 1 & 0 & 2 & 0 & 2 \\ 1 & 0 & 2 & 0 & 1 & 0 & 2 & 0 \\ 0 & 1 & 0 & 1 & 0 & 2 & 0 & 2\end{array}\right| \quad$ CIE6 $=\left|\begin{array}{llllllll}3 & 0 & 2 & 0 & 0 & 2 & 0 & 0 \\ 0 & 2 & 0 & 1 & 0 & 0 & 1 & 2 \\ 2 & 0 & 3 & 0 & 1 & 1 & 0 & 0 \\ 0 & 1 & 0 & 2 & 0 & 1 & 0 & 1 \\ 0 & 0 & 1 & 0 & 2 & 0 & 1 & 1 \\ 2 & 0 & 1 & 1 & 0 & 3 & 0 & 0 \\ 0 & 1 & 0 & 0 & 1 & 0 & 2 & 2 \\ 0 & 2 & 0 & 1 & 1 & 0 & 2 & 3\end{array}\right|$

CIE7 $=\left|\begin{array}{llllllll}3 & 0 & 1 & 0 & 1 & 0 & 0 & 2 \\ 0 & 2 & 0 & 1 & 0 & 1 & 1 & 1 \\ 1 & 0 & 3 & 0 & 1 & 1 & 1 & 0 \\ 0 & 1 & 0 & 2 & 0 & 1 & 0 & 1 \\ 0 & 1 & 0 & 0 & 2 & 0 & 0 & 1 \\ 0 & 1 & 1 & 1 & 0 & 3 & 2 & 0 \\ 0 & 1 & 1 & 0 & 0 & 2 & 2 & 0 \\ 2 & 1 & 0 & 1 & 1 & 0 & 0 & 3\end{array}\right| \operatorname{CIE} 8=\left|\begin{array}{llllllll}3 & 0 & 2 & 1 & 0 & 0 & 1 & 1 \\ 0 & 3 & 0 & 1 & 1 & 2 & 0 & 1 \\ 2 & 0 & 3 & 1 & 1 & 2 & 0 & 1 \\ 1 & 1 & 0 & 2 & 0 & 1 & 0 & 0 \\ 0 & 1 & 1 & 0 & 2 & 1 & 0 & 0 \\ 0 & 2 & 0 & 1 & 1 & 3 & 1 & 0 \\ 1 & 0 & 1 & 0 & 0 & 1 & 2 & 0 \\ 1 & 1 & 1 & 0 & 0 & 0 & 0 & 2\end{array}\right|$

$$
\text { CIE9 }=\left|\begin{array}{llllllll}
2 & 0 & 1 & 0 & 0 & 0 & 2 & 1 \\
0 & 3 & 0 & 1 & 0 & 2 & 0 & 2 \\
1 & 0 & 3 & 0 & 2 & 0 & 2 & 0 \\
0 & 1 & 0 & 2 & 0 & 2 & 0 & 0 \\
0 & 0 & 2 & 0 & 2 & 0 & 1 & 0 \\
0 & 2 & 0 & 2 & 0 & 3 & 0 & 1 \\
2 & 0 & 2 & 0 & 1 & 0 & 3 & 0 \\
0 & 2 & 0 & 0 & 0 & 1 & 0 & 2
\end{array}\right|
$$

As explained in section II the sum of all elements of the CI matrix reflects a unique number that groups the chains of same link assortment. The chains of the link assortment [2(4)-3(4)] are shown in fig-1 have the same group identification number i.e. 52 obtained by summing all the elements of any of the matrices from
CIE1 to CIE9 each matrix will give the same number. Hence the GIDN for this group of chains is 52.The number of automorphs in the chains of GIDN-52 can be determined using the method explained in the section III.

Now consider the chain E1 for the determination of automorphs. The matrix CIE1 is used and the diagonal elements are replaced by zero one by one corresponding to the link of the chain chosen to be the fixed link and the Eigen values of the matrix are determined.table-1 shows the Eigen values for the chain E1. The LSI values for chain E1 shows that the links 1,3,7 \& 8 are the automorphs hence the mechanism developed by these links are not different. Similarly the links 2, 4, 5\&6 shows automorphism hence mechanisms developed by these links will be same. So the chain E1 only gives two mechanisms and two groups of isomorphs each contains four similar mechanisms.

The method discussed in section III is applied on all the chains of the GIDN-52 i.e. from chains E1 to E9 and the values of LSI for the links of the chains are shown in table-2. Chain E2 have similar LSI values for links 1 $\& 6,2 \& 5,3 \& 8$ and $4 \& 7$, thus chain E2 has four groups of automorphs so only four different mechanisms can be obtained.table-2 shows that the chain E3 links1\&5, 2\&4 have same values of LSI and links 3,6,7,9 have different values which indicates that chain E3 gives two groups of automorphs each contains two identical mechanisms hence chain E3 will give 6 different mechanisms. Chain E4 have 4 groups of automorphs each contains two similar mechanisms as shown by LSI values in the table2 hence only four different mechanisms are possible from chain E4. Chain E5 is having two groups of automorphs containing four similar mechanisms hence only two mechanisms are possible from chain E5. Chain E6 have 3 groups of isomorphs contains 2 identical mechanisms, hence only 5 mechanisms are possible from this chain. Chain E7 is free from automorphism. Hence contains 8 different mechanisms. Chain E8 and E9 are having two groups of automorphs each containing four similar mechanisms hence only two mechanisms are possible from chains E8 and E9.

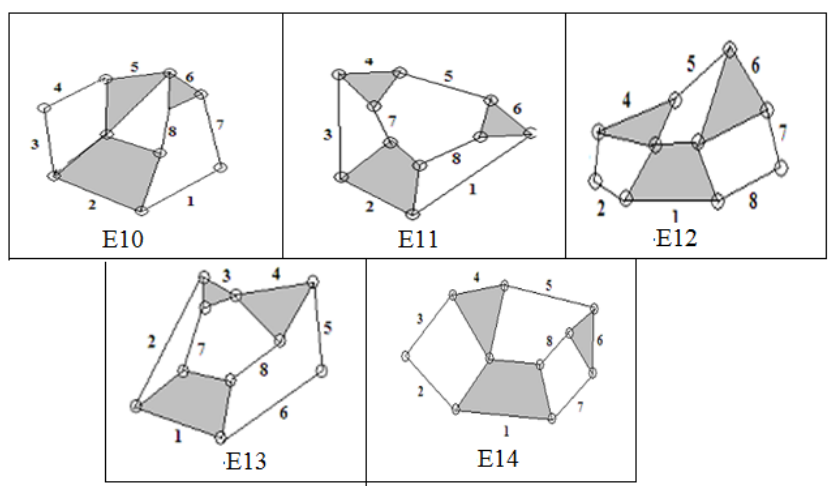

Fig.2 Eight link chains with assortment [2(5)-3(2)-4(1)] 
Table 1. Eigen and LSI values for chain E1

\begin{tabular}{|c|c|c|c|c|c|c|c|c|c|}
\hline $\begin{array}{c}\text { Link } \\
\text { no. } \downarrow\end{array}$ & \multicolumn{7}{|c|}{ Eigen values } & LSI \\
\hline 1 & -1.0221 & 0.5760 & 1.0000 & 1.0000 & 2.2355 & 3.7828 & 4.0000 & 6.4278 & 20.0442 \\
\hline 2 & -0.9228 & -0.0000 & 1.0000 & 1.0000 & 2.2355 & 3.4007 & 4.0000 & 6.2946 & 18.8456 \\
\hline 3 & -1.0221 & 0.5760 & 1.0000 & 1.0000 & 2.2275 & 3.7828 & 4.0000 & 6.4278 & 20.0442 \\
\hline 4 & -0.9228 & -0.0000 & 1.0000 & 1.0000 & 2.2275 & 3.4007 & 4.0000 & 6.2946 & 18.8456 \\
\hline 5 & -0.9228 & -0.0000 & 1.0000 & 1.0000 & 2.2275 & 3.4007 & 4.0000 & 6.2946 & 18.8456 \\
\hline 6 & -0.9228 & -0.0000 & 1.0000 & 1.0000 & 2.2275 & 3.4007 & 4.0000 & 6.2946 & 18.8456 \\
\hline 7 & -1.0221 & 0.5760 & 1.0000 & 1.0000 & 2.2355 & 3.7828 & 4.0000 & 6.4278 & 20.0442 \\
\hline 8 & -1.0221 & 0.5760 & 1.0000 & 1.0000 & 2.2355 & 3.7828 & 4.0000 & 6.4278 & 20.0442 \\
\hline
\end{tabular}

Table 2. LSI values for chains E1 to E9

\begin{tabular}{|c|c|c|c|c|c|c|c|c|}
\hline chain no & link-1 & link-2 & link-3 & link-4 & link-5 & link-6 & link-7 & link-8 \\
\hline E1 & 20.0442 & 18.8456 & 20.0442 & 18.8456 & 18.8456 & 18.8456 & 20.0442 & 20.0442 \\
\hline E2 & 19.0292 & 19.1515 & 20.1500 & 20.2103 & 19.1515 & 19.0292 & 20.2103 & 20.1500 \\
\hline E3 & 19.6314 & 19.3264 & 20.3775 & 19.3264 & 19.6314 & 18.6224 & 19.9743 & 19.9516 \\
\hline E4 & 20.3164 & 20.1450 & 19.7029 & 20.6581 & 19.7029 & 20.1450 & 20.3164 & 20.6581 \\
\hline E5 & 19.1825 & 19.1825 & 20.5498 & 19.1825 & 19.1825 & 20.5498 & 20.5498 & 20.5498 \\
\hline E6 & 20.0702 & 20.3205 & 19.5745 & 19.7527 & 19.7527 & 19.5745 & 20.3205 & 20.7034 \\
\hline E7 & 19.6038 & 19.7650 & 18.9808 & 19.7824 & 19.2459 & 20.0148 & 20.4923 & 20.0494 \\
\hline E8 & 19.9503 & 19.9503 & 19.9503 & 19.7025 & 19.7025 & 19.9503 & 19.7025 & 19.7025 \\
\hline E9 & 20.1898 & 20.2530 & 20.2530 & 20.1898 & 20.1898 & 20.2530 & 20.2530 & 20.1898 \\
\hline
\end{tabular}

CI matrices for the chains E10 to E14 are developed in the similar manner as for the chains E1 to E9 and the GIDN for these chains is determined which comes out to be 54 and the LSI values are also determined which are shown in table-3. The LSI values of chain E10 shows this chain doesn't shows automorphism hence 8 distinct mechanisms will be obtained. Chain E11 has two groups of automorphs one group contains four similar mechanisms and the other two similar mechanisms hence this chain will give four different mechanisms. Chain E12 is having three groups of automorphs containing two similar mechanisms in each group, so five different mechanisms are possible from this chain. Chain E13 and E14 both contain only one group of automorphs contains two similar mechanisms, hence seven different mechanisms are possible from these chains.

Table 3. LSI values for chains E10 to E14

\begin{tabular}{|c|c|c|c|c|c|c|c|c|}
\hline chain no & link-1 & link-2 & link-3 & link-4 & link-5 & link-6 & link-7 & link-8 \\
\hline E10 & 19.7163 & 19.6647 & 20.2945 & 19.7998 & 20.5729 & 19.3773 & 19.737 & 20.3504 \\
\hline E11 & 20.6088 & 18.9282 & 20.6088 & 18.6738 & 19.2111 & 18.6738 & 20.6088 & 20.6088 \\
\hline E12 & 20.3993 & 20.1903 & 20.18 & 20.2005 & 19.746 & 20.2005 & 20.18 & 20.1903 \\
\hline E13 & 18.7201 & 20.6565 & 19.3611 & 18.789 & 19.3818 & 19.5152 & 20.6565 & 20.2173 \\
\hline E14 & 19.8764 & 20.3257 & 20.2069 & 20.0025 & 19.7375 & 19.623 & 20.6743 & 20.6743 \\
\hline
\end{tabular}

Table 4. LSI values for chains E15 to E16

\begin{tabular}{|c|c|c|c|c|c|c|c|c|}
\hline chain no & link-1 & link-2 & link-3 & link-4 & link-5 & link-6 & link-7 & link-8 \\
\hline E15 & 18.6558 & 19.564 & 19.564 & $\mathbf{1 8 . 6 5 5 8}$ & $\mathbf{1 9 . 5 6 4}$ & 19.564 & $\mathbf{2 0 . 8 0 9 7}$ & $\mathbf{2 0 . 8 0 9 7}$ \\
\hline E16 & 19.7047 & 20.000 & 19.7047 & 19.7047 & 20.000 & 19.7047 & 19.7047 & 19.7047 \\
\hline
\end{tabular}




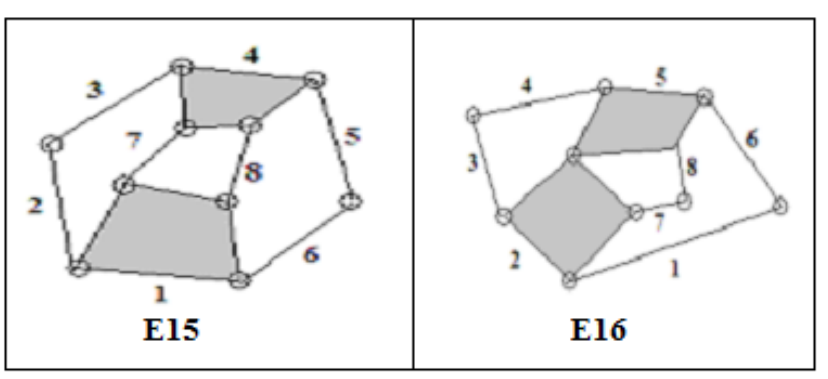

Fig.3 Eight link chains with assortment [2(6)-4(2)]

Fig-3 shows two chains E15 and E16 having link assortment [2(6)-4(2)], the CI matrices of the chains are obtained to find the GIDN of the chains is determined which comes out to be 56. Using the method explained in section III the invariant LSI is obtained for both the chains and their values are listed in table-4. Chain E15 contains three groups of automorphs $(1,4),(2,3,5 \& 6)$ and $(7,8)$ hence this chain develops only three different mechanisms. Chain E16 contains two groups of $(1,3,4,6,7,8)$ and $(2,5)$ hence only two different mechanisms are possible.

\section{RESULTS}

Table 5. Summery of results

\begin{tabular}{|c|c|c|c|}
\hline GIDN & Chain No & $\begin{array}{c}\text { Automorphs } \\
\text { Groups }\end{array}$ & $\begin{array}{c}\text { Distinct } \\
\text { Mechanisms }\end{array}$ \\
\hline \multirow{9}{*}{52} & E1 & 2 & 2 \\
\hline & E2 & 4 & 4 \\
\hline & $\mathbf{E 3}$ & 2 & 6 \\
\hline & E4 & 4 & 4 \\
\hline & E5 & 2 & 2 \\
\hline & E6 & 3 & 5 \\
\hline & E7 & 0 & 8 \\
\hline & E8 & 2 & 2 \\
\hline & E9 & 2 & 2 \\
\hline \multirow{5}{*}{54} & E10 & 0 & 8 \\
\hline & E11 & 2 & 4 \\
\hline & E12 & 3 & 5 \\
\hline & E13 & 1 & 7 \\
\hline & E14 & 1 & 7 \\
\hline \multirow{2}{*}{56} & E15 & 3 & 3 \\
\hline & E16 & 2 & 2 \\
\hline
\end{tabular}

\section{DISCUSSION}

The results depicted in the table 5 shows that the eight link chains are divided into three groups i.e. group 52, 54 and 56. Chains from E1 to E9 belongs to group 52 chains which develops 35 different mechanisms and 21 groups of automorphs. Chains from E10 to E14 belongs to group 54 which develops 31 different mechanisms and 7 groups of automorphs. Chains E15 to E16 belongs to group 56 which contains 5 distinct mechanisms and 5 groups of automorphs.

Hence the total numbers of distinct mechanisms developed by the 16 eight links chains are 71 and 33 group of automorphs.

\section{CONCLUSION}

The categorization of eight link chains done in this paper is successful in assigning a different identification group number termed as GIDN which is the aim of the study, to the chains having different link assortments and also the number of automorphs groups and distinct mechanisms obtained from these chains. All the three things are obtained with the help of the unique matrix representation of theses kinematic chains. Further this method developed in this paper can be applied to develop distinct mechanisms and knowing the number of automorphs groups from the chains with higher number links i.e. chains with $9,10,12$ or even more number of links. The worth of this method is clear with the fact that the number of distinct mechanisms obtained for the eight link chains is 71 which is supported previous research of the author[11,12,13].

\section{REFERENCES}

[1] Franke, R. Vom Aufbau der Getribe: VDI Verlag, 2nd edition. 1958; 1 .

[2] Crossley F.R.. On an Unpublished Work of ALT. J. of Mechanism. 1966; 1:165-170.

[3] Reuleaux F.The Kinematics of Machinery. Macmillan And Co. London. 1876.

[4] Mruthyunjaya T. S. A Computerized Methodology for Structural Synthesis of Kinematic Chains, Part - 1, Formulation, Mech. Mach. Theory. 1984; 19(6):487495.

[5] Mruthyunjaya T. S. A Computerized Methodology for Structural Synthesis of Kinematic Chains, Part - 2, Application to several fully or Partial Known Cases, Mech. Mach. Theory.1984; 19(6):497-505.

[6] Mruthyunjaya T.S. A computerized methodology for structural synthesis of kinematic chains: Part 3: Application to new case of 10-link three freedom chains. Mechanism and Machine Theory. 1984; 19: 507-530.

[7] Agrawal V.P. and Rao J.S. The Mobility Properties of Kinematic Chains. Mech. Mach. Theory.1987; 22:497504.

[8] Sethi V. K. and Agrawal V. P. Hierarchical 
International Journal of Engineering Research and Technology. ISSN 0974-3154 Vol.13, No.3 (2020), pp. 586-591

(C) International Research Publication House. https://dx.doi.org/10.37624/IJERT/13.3.2020.586-591

Classification of Kinematic Chains - A Multigraph Approach. Mech. Mach. Theory.1993; 28:601-614.

[9] Ting K.L. and Dou X., Classification and Branch Identification of Stephenson Six Bar Chains. Mech. Mach. Theory. 1996; 31:283-295.

[10] Rizvi, Syed Shane Haider, Ali Hasan, and R. A. Khan. New Matrix Representation of Kinematic Chains and Determination of Isomorphism. Sch. J. Eng. Tech. 2014;2(4B):561-565.

[11] Rizvi, Syed Shane Haider, Ali Hasan, and R. A. Khan. A New Method Based On the Comparison of the Unique Chain Code to Detect Isomorphism among Kinematic Chains. International Journal of Modern Engineering Research. 2014;1(4): 16-21.
[12] Rizvi, Syed Shane Haider, Ali Hasan, and R. A. Khan. A new method for distinct inversions and isomorphism detection in kinematic chains. Int. J. Mechanisms and Robotic Systems, 2016; 3(1):48-59.

[13] Rizvi, Syed Shane Haider, Ali Hasan, and R. A. Khan. An efficient algorithm for distinct inversions and isomorphism detection in kinematic chains. Elsever Journal Perspectives in Science. 2016; 8:251-252 